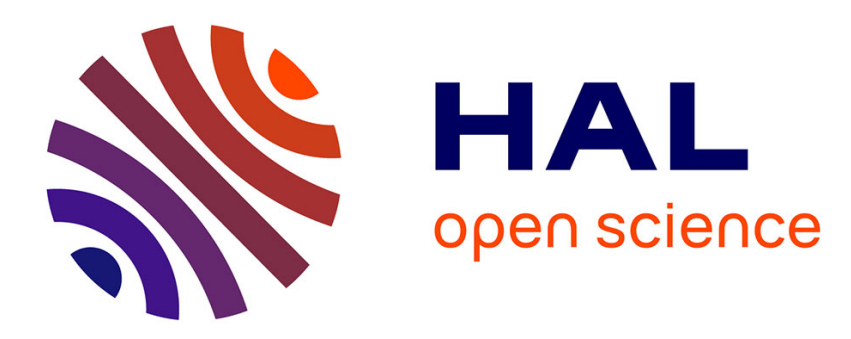

\title{
Convexity of Bertrand oligopoly TU-games with differentiated products
}

Aymeric Lardon

\section{To cite this version:}

Aymeric Lardon. Convexity of Bertrand oligopoly TU-games with differentiated products. 2018. halshs-00544056v2

\section{HAL Id: halshs-00544056 \\ https://shs.hal.science/halshs-00544056v2}

Submitted on 6 Apr 2018

HAL is a multi-disciplinary open access archive for the deposit and dissemination of scientific research documents, whether they are published or not. The documents may come from teaching and research institutions in France or abroad, or from public or private research centers.
L'archive ouverte pluridisciplinaire HAL, est destinée au dépôt et à la diffusion de documents scientifiques de niveau recherche, publiés ou non, émanant des établissements d'enseignement et de recherche français ou étrangers, des laboratoires publics ou privés. 


\title{
Convexity of Bertrand oligopoly TU-games with differentiated products
}

\author{
Aymeric Lardon*广
}

April 6, 2018

\begin{abstract}
We consider Bertrand oligopoly TU-games with differentiated products. We assume that the demand system is Shubik's and that firms operate at a constant and identical marginal and average cost. Our main results state that Bertrand oligopoly TU-games in $\alpha, \beta$ and $\gamma$-characteristic function form satisfy the convexity property, meaning that there exist strong incentives for large-scale cooperation between firms on prices.
\end{abstract}

Keywords: Bertrand competition; Cooperation; Core; Convexity

JEL Classifications: C71, D43

\section{Introduction}

Usually, oligopolies are modeled by means of non-cooperative games. However, in some oligopoly situations firms don't always behave non-cooperatively and if sufficient communication is feasible it may be possible for firms to sign agreements. A question is then whether it is possible for firms to cooperate all together and coordinate their output or price decision. To this end, a cooperative approach for oligopoly situations can be considered by converting the normal form oligopoly game into a cooperative oligopoly game with transferable utility (henceforth, oligopoly TU-game) in which firms can form coalitions acting as a single player. Since every individual profit function depends on each firm's decision, the profit of a coalition depends on outsiders' behavior. As a consequence, the determination of the profit that a coalition can obtain requires to specify how firms outside react. Aumann (1959) proposes two approaches: according to the first, every coalition computes the total profit which it can guarantee itself regardless of what outsiders do; the second approach consists in computing the minimal profit for which outsiders can prevent the firms in the coalition from getting more. These two assumptions lead to consider the $\alpha$ and $\beta$-characteristic functions, respectively. Another approach is proposed by Hart and Kurz (1983), and more specifically by Chander

\footnotetext{
*Université Côte d'Azur, CNRS, GREDEG, France. e-mail: aymeric.lardon@unice.fr

${ }^{\dagger}$ I wish to thank Theo Driessen, Dongshuang Hou, Philippe Solal, Sylvain Béal, Pascal Billand and Christophe Bravard for providing numerous suggestions that substantially improved the exposition of the article.
} 
and Tulkens (1997), where external firms choose their strategy individually as a best reply to the coalitional action. This leads to consider the "partial agreement characteristic function" or, for short, the $\gamma$-characteristic function.

An appropriate set-valued solution for oligopoly TU-games that deals with the possibility for firms to cooperate all together is the core (Shapley 1955). Given a payoff vector in the core, the grand coalition, i.e. the coalition comprising all firms, could form and distribute its worth as payoffs to its members in such a way that no coalition can contest this sharing by breaking off from the grand coalition. In oligopoly TU-games, the stability of cooperation sustained by the grand coalition is then related to the non-emptiness of the core. The convexity property (Shapley 1971) is a sufficent condition for the core to be non-empty and provides a natural way to formalize the idea that it is worthwhile for a player to join larger coalitions. Precisely, it means that the marginal contribution of a player to some coalition increases if the coalition which he joins becomes larger, meaning that there are strong incentives for large-scale cooperation in these game types.

Until now, many works have dealt with Cournot oligopoly TU-games. With or without transferable technologies, ${ }^{1}$ Zhao $(1999 a, b)$ shows that the $\alpha$ and $\beta$-characteristic functions lead to the same class of Cournot oligopoly TU-games. When technologies are transferable, Zhao (1999a) provides a necessary and sufficient condition to establish the convexity property in case the inverse demand function and cost functions are linear. Although these games may fail to be convex in general, Norde et al. (2002) show they are nevertheless totally balanced. When technologies are not transferable, Zhao (1999b) proves that the core of such games is non-empty if every individual profit function is continuous and concave. Furthermore, Norde et al. (2002) show that these games are convex in case the inverse demand function and cost functions are linear. For the class of Cournot oligopoly TU-games in $\gamma$-characteristic function form, Lardon (2012) shows that the differentiability of the inverse demand function ensures that these games are well-defined and provides two core existence results. The first result establishes that such games are balanced, and therefore have a non-empty core, if every individual profit function is concave. When cost functions are linear, the second result provides a single-valued allocation rule in the core, called NP(Nash Pro rata)-value, which is characterized by four axioms: efficiency, null firm, monotonicity and non-cooperative fairness. ${ }^{2}$ Lardon (2017) proposes to extend this setting and study the core of interval oligopoly games under the $\gamma$-approach where each coalition is endowed with an interval of possible worths. In quantity competition, Driessen et al. (2017) have also dealt with Stackelberg oligopoly TU-games in $\gamma$-characteristic function form where each coalition acts as a leader and outsiders play as followers. ${ }^{3}$ They provide a necessary and sufficient condition, depending on the heterogeneity of costs, for the non-emptiness of the core. Another interesting approach is due to Lekeas and Stamatopoulos (2014) where each coalition cannot accurately predict outsiders' behavior and assigns various probability distributions over the set of possible partitions.

Unfortunately, few works have dealt with Bertrand oligopoly TU-games. Kaneko (1978)

\footnotetext{
${ }^{1}$ We refer to Norde et al. (2002) for a detailed discussion of this distinction.

${ }^{2}$ Lardon (2012) gives a precise description of these axioms.

${ }^{3}$ Thereupon, Julien (2017) studies the existence and uniqueness of a Stackelberg equilibrium with multiple leaders and followers.
} 
considers a Bertrand oligopoly situation where a finite number of firms sell a homogeneous product to a continuum of consumers. Kaneko assumes that a subset of firms and consumers can cooperate by trading the good among themselves. The main result establishes that the core is empty when there are more than two firms. Deneckere and Davidson (1985) consider a Bertrand oligopoly situation with differentiated products in which the demand system is Shubik's (1980) and firms operate at a constant and identical marginal and average cost. They study the equilibrium distribution of prices and profits among coalitions and show that a merger of two coalitions implies that all the firms charge higher prices, and so benefits all the industry. They also prove that these games have a superadditive property in the sense that a merger of two disjoint coalitions results in a joint after-merger profit for them which is greater than the sum of their pre-merger profits. For the same model, Huang and Sjöström (2003) provide a necessary and sufficient condition for the non-emptiness of the recursive core $^{4}$ which requires that the substitutability parameter must be greater than or equal to some number that depends on the size of the industry. They conclude that the recursive core is empty when there are more than ten firms.

As a counterpart to this lack of interest in the study of Bertrand oligopoly TU-games, ${ }^{5}$ we define Bertrand oligopoly TU-games by considering successively the $\alpha, \beta$ and $\gamma$-characteristic functions as described above. As for Cournot oligopoly TU-games, we show that the $\alpha$ and $\beta$-characteristic functions lead to the same class of Bertrand oligopoly TU-games. Our main results state that the convexity property holds for these classes of games. These findings extend the superadditivity result of Deneckere and Davidson (1985) and contrasts sharply with the negative core existence results of Kaneko (1978) and Huang and Sjöström (2003). Moreover, an important distinction between Cournot and Bertrand competition is that the former has strategic substitutabilities and the latter has strategic complementarities. Thus, although Cournot and Bertrand oligopoly games are basically different in their non-cooperative forms, it appears that their cooperative forms may have the same core geometrical structure. ${ }^{6}$

The remainder of the article is structured as follows. In Section 2 we introduce the model and some notations. Section 3 establishes that Bertrand oligopoly TU-games in $\alpha, \beta$ and $\gamma$-characteristic function form are convex. Section 4 gives some concluding remarks. Lastly, Section 5 is the appendix where proofs of all the results are presented.

\section{The model}

We consider a Bertrand oligopoly situation $\left(N,\left(D_{i}, C_{i}\right)_{i \in N}\right)$ where $N=\{1,2, \ldots, n\}$ is the finite set of firms, and $D_{i}: \mathbb{R}_{+}^{n} \longrightarrow \mathbb{R}$ and $C_{i}: \mathbb{R}_{+} \longrightarrow \mathbb{R}_{+}, i \in N$, are firm $i$ 's demand and cost functions, respectively. Throughout this article, we assume that:

(a) the demand system is Shubik's (1980):

\footnotetext{
${ }^{4}$ The worth of a coalition is defined in a recursive procedure applying the core solution concept to a "reduced game" in order to predict outsiders' behavior.

${ }^{5}$ When technologies are transferable, Lardon (2014) studies the core of Bertrand oligopoly TU-games in $\alpha$ and $\beta$-characteristic function form.

${ }^{6}$ The convexity property ensures that the core is the convex combination of the marginal vectors (Shapley 1971).
} 


$$
\forall i \in N, D_{i}\left(p_{1}, \ldots, p_{n}\right)=V-p_{i}-r\left(p_{i}-\frac{1}{n} \sum_{j \in N} p_{j}\right)
$$

where $p_{j}$ is the price charged by firm $j, V \in \mathbb{R}_{+}$is the intercept of demand and $r \in \mathbb{R}_{++}$is the substitutability parameter. ${ }^{7}$ The quantity demanded of firm $i$ 's brand depends on its own price $p_{i}$ and on the difference between $p_{i}$ and the average price in the industry $\sum_{j \in N} p_{j} / n$. This quantity is decreasing with respect to $p_{i}$ and increasing with respect to $p_{j}, j \neq i$;

(b) firms operate at a constant and identical marginal and average cost:

$$
\forall i \in N, C_{i}(x)=c x,
$$

where $c \in \mathbb{R}_{+}$is firm i's marginal and average cost, and $x \in \mathbb{R}_{+}$is the quantity demanded of firm $i$ 's brand.

Given assumptions (a) and (b), a Bertrand oligopoly situation is summarized by the 4 -tuple $(N, V, r, c)$.

The normal form Bertrand oligopoly game $\left(N,\left(X_{i}, \pi_{i}\right)_{i \in N}\right)$ associated with the Bertrand oligopoly situation $(N, V, r, c)$ is defined as follows:

1. the set of firms is $N=\{1, \ldots, n\}$;

2. for every $i \in N$, the individual strategy set is $X_{i}=\mathbb{R}_{+}$where $p_{i} \in X_{i}$ represents the price charged by firm $i$; the set of strategy profiles is $X_{N}=\prod_{i \in N} X_{i}$ where $p=\left(p_{i}\right)_{i \in N}$ is a representative element of $X_{N}$;

3. for every $i \in N$, the individual profit function $\pi_{i}: X_{N} \longrightarrow \mathbb{R}$ is defined as:

$$
\pi_{i}(p)=D_{i}(p)\left(p_{i}-c\right)
$$

As mentioned above, we want to analyze the stability of coalitions and the incentives for cooperation in Bertrand competition. To this end, we have to convert the normal form Bertrand oligopoly game $\left(N,\left(X_{i}, \pi_{i}\right)_{i \in N}\right)$ into a Bertrand oligopoly TU-game which is the purpose of Section 3. In the remainder of this section, we give the definitions of a general TU-game and the core and we introduce the notions of superadditivity and convexity. We denote by $2^{N}$ the power set of $N$ and call a subset $S \in 2^{N}, S \neq \emptyset$, a coalition. The size $s=|S|$ of coalition $S$ is the number of players in $S$. A TU-game $(N, v)$ is a set function $v: 2^{N} \longrightarrow \mathbb{R}$ with the convention that $v(\emptyset)=0$, which assigns a number $v(S) \in \mathbb{R}$ to every coalition $S \in 2^{N}$. The number $v(S)$ is the worth of coalition $S$. For a fixed set of players $N$, we denote by $G^{N}$ the set of TU-games where $v$ is a representative element of $G^{N}$. In a TU-game $v \in G^{N}$, every player $i \in N$ may receive a payoff $\sigma_{i} \in \mathbb{R}$. A vector

\footnotetext{
${ }^{7}$ When $r$ approaches zero, products become unrelated, and when $r$ approaches infinity, products become perfect substitutes.
} 
$\sigma=\left(\sigma_{1}, \ldots, \sigma_{n}\right)$ is a payoff vector. We say that a payoff vector $\sigma \in \mathbb{R}^{n}$ is acceptable if for every coalition $S \in 2^{N}, \sum_{i \in S} \sigma_{i} \geq v(S)$, i.e. the payoff vector provides a total payoff to the members of coalition $S$ that is at least as great as its worth. We say that a payoff vector $\sigma \in \mathbb{R}^{n}$ is efficient if $\sum_{i \in N} \sigma_{i}=v(N)$, i.e. the payoff vector provides a total payoff to all the players that is equal to the worth of the grand coalition $N$. The core (Shapley 1955) of a TU-game $v \in G^{N}$ is the set $C(v)$ of payoff vectors that are both acceptable and efficient:

$$
C(v)=\left\{\sigma \in \mathbb{R}^{n}: \forall S \in 2^{N}, \sum_{i \in S} \sigma_{i} \geq v(S) \text { and } \sum_{i \in N} \sigma_{i}=v(N)\right\} .
$$

Given a payoff vector in the core, the grand coalition can form and distribute its worth as payoffs to its members in such a way that no coalition can contest this sharing by breaking off from the grand coalition.

A TU-game $v \in G^{N}$ is superadditive if:

$$
\forall S, T \in 2^{N} \text { such that } S \cap T=\emptyset, v(S)+v(T) \leq v(S \cup T) .
$$

The notion of a convex TU-game is introduced by Shapley (1971) and provides a natural way to formalize the idea that it is worthwhile for a player to join larger coalitions. A TU-game $v \in G^{N}$ is convex if one of the following two equivalent conditions is satisfied: ${ }^{8}$

$$
\forall S, T \in 2^{N}, v(S)+v(T) \leq v(S \cup T)+v(S \cap T)
$$

or equivalently:

$$
\forall i, j \in N, \forall S \subseteq N \backslash\{i, j\}, v(S \cup\{i\})-v(S) \leq v(S \cup\{i, j\})-v(S \cup\{j\})
$$

Observe that every convex TU-game is superadditive. The convexity property means that the marginal contribution of a player to some coalition increases if the coalition which he joins becomes larger. Thus, the convexity property can be regarded as a strong incentive for large-scale cooperation in a TU-game.

\section{Convexity of Bertrand oligopoly TU-games}

\subsection{Bertrand oligopoly TU-games in $\alpha$ and $\beta$-characteristic function form}

In this section, we convert a normal form Bertrand oligopoly game into a Bertrand oligopoly TU-game in such a way that every coalition has pessimistic expectations on its feasible profits. Traditionally, there are two main ways of converting a normal form game into a cooperative game called game in $\alpha$ and $\beta$-characteristic function form, respectively (Aumann 1959). In the first case, the worth of a coalition is obtained by computing the profit which its members can guarantee themselves regardless of what outsiders do. In the second case, the worth of a coalition can be derived by computing the minimal profit such that outsiders can prevent

\footnotetext{
${ }^{8}$ The proof of this result is given by Shapley (1971).
} 
its members from getting more. First, we show that the $\alpha$ and $\beta$-characteristic functions are well-defined and lead to the same class of Bertrand oligopoly TU-games. This equality between the $\alpha$ and $\beta$-characteristic functions is a useful property, as it relieves us of the burden of choosing between the $\alpha$ and $\beta$-characteristic functions when describing collusive profits. Then, we prove that the convexity property holds for this class of games, i.e. when every coalition has pessimistic expectations on its feasible profits there exist strong incentives for firms to cooperate all together.

In order to define the $\alpha$ and $\beta$-characteristic functions, we denote by $X_{S}=\prod_{i \in S} X_{i}$ the coalition strategy set of coalition $S \in 2^{N}$ and $X_{N \backslash S}=\prod_{i \in N \backslash S} X_{i}$ the set of outsiders' strategy profiles for which $p_{S}=\left(p_{i}\right)_{i \in S}$ and $p_{N \backslash S}=\left(p_{i}\right)_{i \in N \backslash S}$ are the representative elements, respectively. For every coalition $S \in 2^{N}$, the coalition profit function $\pi_{S}$ : $X_{S} \times X_{N \backslash S} \longrightarrow \mathbb{R}$ is defined as:

$$
\pi_{S}\left(p_{S}, p_{N \backslash S}\right)=\sum_{i \in S} \pi_{i}(p) .
$$

Given the normal form Bertrand oligopoly game $\left(N,\left(X_{i}, \pi_{i}\right)_{i \in N}\right)$, the $\alpha$ and $\beta$-characteristic functions are defined for every coalition $S \in 2^{N}$ as:

$$
v_{\alpha}(S)=\max _{p_{S} \in X_{S}} \min _{p_{N} \backslash S} \in X_{N \backslash S} \pi_{S}\left(p_{S}, p_{N \backslash S}\right)
$$

and

$$
v_{\beta}(S)=\min _{p_{N \backslash S} \in X_{N \backslash S}} \max _{p_{S} \in X_{S}} \pi_{S}\left(p_{S}, p_{N \backslash S}\right)
$$

respectively. For a fixed set of firms $N$, we denote by $G_{o}^{N} \subseteq G^{N}$ the set of Bertrand oligopoly TU-games.

The following proposition states that Bertrand oligopoly TU-games in $\beta$-characteristic function form are well-defined.

Proposition 3.1 Let $\left(N,\left(X_{i}, \pi_{i}\right)_{i \in N}\right)$ be a normal form Bertrand oligopoly game. Then, for every coalition $S \in 2^{N}$, it holds that:

$$
v_{\beta}(S)=\pi_{S}\left(\bar{p}_{S}, \bar{p}_{N \backslash S}\right),
$$

where $\left(\bar{p}_{S}, \bar{p}_{N \backslash S}\right) \in X_{S} \times X_{N \backslash S}$ is given by:

$$
\forall i \in S, \bar{p}_{i}=\max \left\{c, \frac{V}{2(1+r(n-s) / n)}+\frac{c}{2}\right\}
$$

and

$$
\sum_{j \in N \backslash S} \bar{p}_{j}=\max \left\{0, \frac{n}{r}\left(c\left(1+r \frac{(n-s)}{n}\right)-V\right)\right\}
$$


Unsurprisingly, a direct consequence of this result is that any Bertrand oligopoly TU-game $v_{\beta} \in G_{o}^{N}$ is symmetric, i.e. the worth of a coalition $S \in 2^{N}$ only depends on its size. ${ }^{9}$ Proposition 3.1 calls for some further comments which will be useful for the sequel.

\section{Remark 3.2}

For every coalition $S \in 2^{N}$, it holds that:

1. If $V \leq c(1+r(n-s) / n)$, then by (4) each member $i \in S$ charges prices equal to their marginal cost, $\bar{p}_{i}=c$, and by (5) the outsiders charge a non-negative average price, $\sum_{j \in N \backslash S} \bar{p}_{j} /(n-s) \geq 0$. In this case, coalition $S$ obtains a zero profit, $v_{\beta}(S)=0$.

2. If $V>c(1+r(n-s) / n)$, then by (4) each member $i \in S$ charges prices strictly greater than their marginal cost, $\bar{p}_{i}>c$, and by (5) the outsiders charge a zero average price, $\sum_{j \in N \backslash S} \bar{p}_{j} /(n-s)=0$. In this case, coalition $S$ obtains a positive profit, $v_{\beta}(S)>0$.

3. The computation of the worth $v_{\beta}(S)$ is consistent with the fact that the quantity demanded of firm $i$ 's brand, $i \in S$, is positive since for every $i \in S, D_{i}(\bar{p}) \geq 0$.

By solving successively the minimization and the maximization problems defined in (2), we can show that Bertrand oligopoly TU-games in $\alpha$-characteristic function form are also welldefined. The technical arguments are similar to those used in the proof of Proposition 3.1. For general TU-games, Zhao (1999b) shows that the $\alpha$ and $\beta$-characteristic functions are equal when every strategy set is compact, every utility (or profit) function is continuous, and the strong separability condition is satisfied. This condition requires that the utility (or profit) function of any coalition $S$ and each of its members' utility (or profit) functions have the same minimizers. Zhao's result (1999b) holds for the specific class of Bertrand oligopoly TU-games with pessimistic expectations. First, we compactify the strategy sets by assuming that for every firm $i \in N, X_{i}=[0, \mathbf{p}]$ where $\mathbf{p}$ is sufficiently large so that the maximization/minimization problems defined in (2) and (3) have interior solutions. Then, it is clear that every individual profit function $\pi_{i}$ is continuous. Finally, since the demand system is symmetric and firms operate at a constant and identical marginal and average cost, Bertrand oligopoly TU-games satisfy the strong separability condition.

Corollary 3.3 Let $\left(N,\left(X_{i}, \pi_{i}\right)_{i \in N}\right)$ be a normal form Bertrand oligopoly game. Then, for every coalition $S \in 2^{N}$, it holds that:

$$
v_{\alpha}(S)=v_{\beta}(S) .
$$

Corollary 3.3 implies that outsiders' strategy profile $\bar{p}_{N \backslash S}$ that best punishes coalition $S$ as a first mover ( $\alpha$-characteristic function) also best punishes $S$ as a second mover ( $\beta$ characteristic function). We deduce from Corollary 3.3 that for any $v_{\alpha} \in G_{o}^{N}$ and any

\footnotetext{
${ }^{9}$ Formally, a TU-game $v \in G^{N}$ is symmetric if there exists a function $f: \mathbb{N} \longrightarrow \mathbb{R}$ such that for every coalition $S \in 2^{N}, v(S)=f(s)$.
} 
$v_{\beta} \in G_{o}^{N}$ associated with the Bertrand oligopoly situation $(N, V, r, c)$, it holds that $C\left(v_{\alpha}\right)=$ $C\left(v_{\beta}\right)$. The following theorem states that the convexity property holds for the class of Bertrand oligopoly TU-games in $\beta$-characteristic function form.

Theorem 3.4 Every Bertrand oligopoly TU-game $v_{\beta} \in G_{o}^{N}$ is convex.

When firms operate at distinct marginal costs, Driessen et al. (2011) prove that the Shapley value of Bertrand oligopoly TU-games in $\beta$-characteristic function form is fully determined on the basis of linearity applied to an appealing decomposition of these games.

\subsection{Bertrand oligopoly TU-games in $\gamma$-characteristic function form}

In this section, we consider the blocking rule for which outsiders choose their strategy individually as a best reply facing the deviating coalition (Hart and Kurz 1983, Chander and Tulkens 1997). Such an equilibrium is called a partial agreement equilibrium and leads to consider the "partial agreement characteristic function" or, for short, the $\gamma$-characteristic function. First, we verify that the $\gamma$-characteristic function is well-defined. Then, we prove that the convexity property also holds for this class of games.

Given the normal form Bertrand oligopoly game $\left(N,\left(X_{i}, \pi_{i}\right)_{i \in N}\right)$ and a coalition $S \in 2^{N}$, a strategy profile $\left(p_{S}^{*}, \tilde{p}_{N \backslash S}\right) \in X_{S} \times X_{N \backslash S}$ is a partial agreement equilibrium under $S$ if:

$$
\forall p_{S} \in X_{S}, \pi_{S}\left(p_{S}^{*}, \tilde{p}_{N \backslash S}\right) \geq \pi_{S}\left(p_{S}, \tilde{p}_{N \backslash S}\right)
$$

and

$$
\forall j \in N \backslash S, \forall p_{j} \in X_{j}, \pi_{j}\left(p_{S}^{*}, \tilde{p}_{N \backslash S}\right) \geq \pi_{j}\left(p_{S}^{*}, \tilde{p}_{N \backslash(S \cup\{j\})}, p_{j}\right)
$$

The $\gamma$-characteristic function is defined for every coalition $S \in 2^{N}$ as:

$$
v_{\gamma}(S)=\pi_{S}\left(p_{S}^{*}, \tilde{p}_{N \backslash S}\right),
$$

where $\left(p_{S}^{*}, \tilde{p}_{N \backslash S}\right) \in X_{S} \times X_{N \backslash S}$ is a partial agreement equilibrium under $S$.

Throughout this section, in addition to assumptions (a) and (b), we assume that:

(c) the intercept of demand $V \in \mathbb{R}_{+}$is strictly greater than the marginal cost $c \in \mathbb{R}_{+}$.

For every partial agreement equilibrium under $S$, assumption (c) ensures that the quantity demanded of every firm's brand is non-negative.

Deneckere and Davidson (1985) study normal form Bertrand oligopoly games with general coalition structures. A coalition structure $\mathcal{P}$ is a partition of the set of firms $N$, i.e. $\mathcal{P}=\left\{S_{1}, \ldots, S_{k}\right\}, k \in\{1, \ldots, n\}$. An element of a coalition structure, $S \in \mathcal{P}$, is called an admissible coalition in $\mathcal{P}$. We denote by $\Pi(N)$ the set of coalition structures on $N$. We introduce a binary relation $\leq^{F}$ on $\Pi(N)$ defined as follows: we say that a coalition structure 
$\mathcal{P}^{\prime} \in \Pi(N)$ is finer than a coalition structure $\mathcal{P} \in \Pi(N)$ (or $\mathcal{P}$ is coarser than $\mathcal{P}^{\prime}$ ) which we write $\mathcal{P} \leq F \mathcal{P}^{\prime}$ if for every admissible coalition $S$ in $\mathcal{P}^{\prime}$ there exists an admissible coalition $T$ in $\mathcal{P}$ such that $T \supseteq S$. Note that $\left(\Pi(N), \leq^{F}\right)$ is a complete lattice.

Given $\mathcal{P} \in \Pi(N)$, the normal form Bertrand oligopoly game $\left(\mathcal{P},\left(X_{S}, \pi_{S}\right)_{S \in \mathcal{P}}\right)$ associated with the Bertrand oligopoly situation $(N, V, r, c)$ is defined as follows:

1. the set of players (or admissible coalitions) is $\mathcal{P}$;

2. for every $S \in \mathcal{P}$, the coalition strategy set is $X_{S}=\prod_{i \in S} X_{i}$;

3. for every $S \in \mathcal{P}$, the coalition profit function is $\pi_{S}=\sum_{i \in S} \pi_{i}$.

The following proposition contains different results established by Deneckere and Davidson (1985) which will be useful for the sequel.

\section{Proposition 3.5 (Deneckere and Davidson 1985)}

- Let $\mathcal{P} \in \Pi(N)$ be a coalition structure and let $\left(\mathcal{P},\left(X_{S}, \pi_{S}\right)_{S \in \mathcal{P}}\right)$ be the associated normal form Bertrand oligopoly game. Then,

1. there exists a unique Nash equilibrium $p^{*} \in X_{N}$ such that:

$$
\forall S \in \mathcal{P}, \exists p^{* S} \in \mathbb{R}_{+}: \forall i \in S, p_{i}^{*}=p^{* S}
$$

2. it holds that:

$$
\forall S, T \in \mathcal{P}: s \leq t, p^{* S} \leq p^{* T},
$$

with strict inequality if $s<t$.

- Let $\mathcal{P}, \mathcal{P}^{\prime} \in \Pi(N)$ be two coalition structures such that $\mathcal{P} \leq F \mathcal{P}^{\prime}$. Let $p^{*} \in X_{N}$ and $p^{* *} \in X_{N}$ be the unique Nash equilibria of the normal form Bertrand oligopoly games $\left(\mathcal{P},\left(X_{S}, \pi_{S}\right)_{S \in \mathcal{P}}\right)$ and $\left(\mathcal{P}^{\prime},\left(X_{S}, \pi_{S}\right)_{S \in \mathcal{P}^{\prime}}\right)$, respectively. Then,

3. it holds that for every $i \in N, p_{i}^{*} \geq p_{i}^{* *}$.

Point 1 of Proposition 3.5 establishes the existence of a unique Nash equilibrium for every normal form Bertrand oligopoly game $\left(\mathcal{P},\left(X_{S}, \pi_{S}\right)_{S \in \mathcal{P}}\right)$ and stipulates that the members of an admissible coalition $S \in \mathcal{P}$ charge identical prices. Point 2 of Proposition 3.5 characterizes the distribution of prices within a coalition structure and states that if the size $t$ of an admissible coalition $T \in \mathcal{P}$ is greater than or equal to the size $s$ of an admissible coalition $S \in \mathcal{P}$, then the firms in $T$ charge higher prices than the firms in $S$. Point 3 of Proposition 3.5 analyses the variations in equilibrium prices according to the coarseness of the coalition structure and specifies that all the firms charge higher prices when the coalition structure becomes coarser. It follows from Point 1 of Proposition 3.5 that Bertrand oligopoly TUgames in $\gamma$-characteristic function form are well-defined.

Corollary 3.6 Let $\left(N,\left(X_{i}, \pi_{i}\right)_{i \in N}\right)$ be a normal form Bertrand oligopoly game. Then, for every coalition $S \in 2^{N}$, there exists a unique partial agreement equilibrium under $S$. 
By solving the maximization problems derived from (6) and (7), the unique partial agreement equilibrium under $S,\left(p_{S}^{*}, \tilde{p}_{N \backslash S}\right) \in X_{S} \times X_{N \backslash S}$, is given by:

$$
\forall i \in S, p_{i}^{*}=\frac{(V-c)(2 n(1+r)-r) n}{2(2 n+r(n+s-1))(n+r(n-s))-r^{2} s(n-s)}+c
$$

and

$$
\forall j \in N \backslash S, \tilde{p}_{j}=\frac{(V-c)(2 n(1+r)-r s) n}{2(2 n+r(n+s-1))(n+r(n-s))-r^{2} s(n-s)}+c
$$

When $c=0$, Deneckere and Davidson (1985) provide equivalent expressions of (8) and (9) from which we deduce that Bertrand oligopoly TU-games in $\gamma$-characteristic function form are symmetric. More precisely, it follows from (8) that the members of a coalition $S \in 2^{N}$ charge identical prices, i.e. for every $i \in S$, there exists $p^{* s} \in \mathbb{R}_{+}$such that $p_{i}^{*}=p^{* s}$. It also follows from (9) that outsiders charge identical prices, i.e. for every $j \in N \backslash S$, there exists $\tilde{p}^{s} \in \mathbb{R}_{+}$such that $\tilde{p}_{j}=\tilde{p}^{s}$. With these notations in mind, Proposition 3.5 calls for some further comments.

\section{Remark 3.7}

1. For every coalition $S \in 2^{N}$, we deduce from Point 2 of Proposition 3.5 that $p^{* s} \geq \tilde{p}^{s}$, i.e. the members of coalition $S$ charge higher prices than the outsiders.

2. For every coalition $S, T \in 2^{N}$ such that $S \subseteq T$, it follows from Point 3 of Proposition 3.5 that $p^{* s} \leq p^{* t}$ and $\tilde{p}^{s} \leq \tilde{p}^{t}$.

3. For every coalition $S \in 2^{N}$, let $\left(p_{S}^{*}, \tilde{p}_{N \backslash S}\right) \in X_{S} \times X_{N \backslash S}$ be the unique partial agreement equilibrium under $S$. If $p^{* s}>c$ and $\tilde{p}^{s}>c$ then for every $i \in N$, $D_{i}\left(p_{S}^{*}, \tilde{p}_{N \backslash S}\right) \geq 0$. In order to prove this result, for the sake of contradiction, assume that there exists $i \in N$ such that $D_{i}\left(p_{S}^{*}, \tilde{p}_{N \backslash S}\right)<0$, and $p^{* s}>c$ and $\tilde{p}^{s}>c$. We distinguish two cases:

- if $i \in S$ then we deduce from Point 1 of Proposition 3.5 that for every $j \in S$, $D_{j}\left(p_{S}^{*}, \tilde{p}_{N \backslash S}\right)=D_{i}\left(p_{S}^{*}, \tilde{p}_{N \backslash S}\right)<0$. Hence, it follows from $p^{* s}>c$ that coalition $S$ obtains a negative profit.

- if $i \in N \backslash S$ then it follows from $\tilde{p}^{s}>c$ that outsider $i$ obtains a negative profit.

In both cases, since coalition $S$ or every outsider can guarantee a non-negative profit by charging $p^{* S}=c$ or $\tilde{p}^{s}=c$, respectively, we conclude that $\left(p_{S}^{*}, \tilde{p}_{N \backslash S}\right) \in X_{S} \times X_{N \backslash S}$ is not a partial agreement equilibrium under $S$, a contradiction.

By (8) and (9), note that $p^{* s}>c$ and $\tilde{p}^{s}>c$ is satisfied if and only if $V>c$, which corresponds to assumption (c). Thus, assumption (c) ensures that the quantity demanded of every firm's brand is non-negative. 
It seems clear that for every coalition $S \in 2^{N} \backslash\{N\}, v_{\gamma}(S) \leq v_{\beta}(S)$ and $v_{\gamma}(N)=v_{\beta}(N)$, so that the core associated with the $\gamma$-characteristic function is included in the core associated with the $\beta$-characteristic function ${ }^{10}$ as illustrated in the following example.

Example 3.8 Consider the Bertrand oligopoly situation $(N, V, r, c)$ where $N=\{1,2,3\}$, $V=5, r=2$ and $c=1$. For every coalition $S \in 2^{N}$, the worths $v_{\beta}(S)$ and $v_{\gamma}(S)$ are given in the following table:

\begin{tabular}{|c|ccccccc|}
\hline$S$ & $\{1\}$ & $\{2\}$ & $\{3\}$ & $\{1,2\}$ & $\{1,3\}$ & $\{2,3\}$ & $\{1,2,3\}$ \\
\hline$v_{\beta}(S)$ & 0.76 & 0.76 & 0.76 & 3.33 & 3.33 & 3.33 & 12 \\
\hline$v_{\gamma}(S)$ & 3.36 & 3.36 & 3.36 & 7.05 & 7.05 & 7.05 & 12 \\
\hline
\end{tabular}

It follows that the cores $C\left(v_{\beta}\right)$ and $C\left(v_{\gamma}\right)$ are given by:

$$
C\left(v_{\beta}\right)=\left\{\sigma \in \mathbb{R}^{3}: \sum_{i \in N} \sigma_{i}=12 \text { and } \forall i \in\{1,2,3\}, 0.76 \leq \sigma_{i} \leq 8.67\right\},
$$

and

$$
C\left(v_{\gamma}\right)=\left\{\sigma \in \mathbb{R}^{3}: \sum_{i \in N} \sigma_{i}=12 \text { and } \forall i \in\{1,2,3\}, 3.36 \leq \sigma_{i} \leq 4.95\right\} .
$$

The 2-simplex below represents these two core configurations:



\footnotetext{
${ }^{10}$ This result is intuitive and holds for general TU-games.
} 
Thus, from the Bertrand oligopoly TU-game $v_{\beta} \in G_{o}^{N}$ to the Bertrand oligopoly TU-game $v_{\gamma} \in G_{o}^{N}$, we see that the core is substantially reduced.

A well-known single-valued allocation rule, ${ }^{11}$ is the Equal Division Solution. For every TU-game $v \in G^{N}$ the Equal Division Solution, denoted by $E D(v)$, is defined as:

$$
\forall i \in N, E D_{i}(v)=\frac{v(N)}{n} .
$$

The Equal Division Solution distributes the worth of the grand coalition equally among the players. Deneckere and Davidson (1985) establishes that any coalition $S \subseteq N$ of size $s \in\{2, \ldots, n\}$ is profitable to each of the merging parties and that outsiders take a free ride and earn larger profits than do insiders. Moreover, they prove that coalitions are increasingly profitable, i.e. for all $s \in\{1, \ldots, n\}$ and for all $t \in\{1, \ldots, n-1$ such that $s>t$, it holds that:

$$
\frac{v_{\gamma}(S)}{s}>\frac{v_{\gamma}(S)}{t}{ }^{12}
$$

This property ensures that the Equal Division Solution belongs to the core. ${ }^{13}$

Corollary 3.9 Any Bertrand oligopoly TU-game $v_{\gamma} \in G_{o}^{N}$ has at least one core element, namely the Equal Division Solution.

The following result goes further and makes precise the sense of profitability of cooperation between firms.

Theorem 3.10 Every Bertrand oligopoly TU-game $v_{\gamma} \in G_{o}^{N}$ is convex.

This result extends the superadditivity property estbalished by Deneckere and Davidson (1985).

\section{Concluding remarks}

In this article, we have considered a fully cooperative approach for oligopoly situations. Our analysis of incentives to cooperate is complementary to the previous theoretical and empirical studies of coalition formation process in the framework of non-cooperative games (see, for example, Bloch 1995 and Levenstein 1997). Generally speaking, we have proved that the convexity property holds for Bertrand oligopoly TU-games in $\alpha, \beta$ and $\gamma$-characteristic function form. This generalizes the superaddivity result in Deneckere and Davidson (1985) and contrasts sharply with the negative core existence results of Kaneko (1978) and Huang

\footnotetext{
${ }^{11}$ A single-valued allocation rule on $G^{N}$ is a mapping $\rho: G^{N} \longrightarrow \mathbb{R}^{n}$ that associates to every TU-game $v \in G^{N}$ a payoff vector $\rho(v) \in \mathbb{R}^{n}$.

${ }^{12}$ Deneckere and Davidson (1985) prove that the profits of the coalitions are superadditive for any market structure.

${ }^{13}$ For general symmetric TU-games, the set of inequalities $v(N) / n \geq v(S) / s$ for any $S \in 2^{N}, S \neq \emptyset$, is a necessary and sufficient condition for the non-emptiness of the core.
} 
and Sjöström (2003). Another approach proposed by Hart and Kurz (1983) consists in considering that for every coalition $S \in 2^{N}$, outsiders will form the complementary coalition $N \backslash S$ and correlate their strategies to maximize their joint profit. This leads to consider the $\delta$-characteristic function. Given the normal form Bertrand oligopoly game $\left(N,\left(X_{i}, \pi_{i}\right)_{i \in N}\right)$ and any coalition $S \in 2^{N}$ the $\delta$-characteristic function is then given by:

$$
v_{\delta}(S)=\frac{s n(V-c)^{2}(2 n+r(n+s))^{2}(n+r(n-s))}{\left(4 n^{2}(1+r)+3 r^{2} s(n-s)\right)^{2}} \cdot 14
$$

In this case, the convexity property does not hold anymore since the core may be empty. Precisely, it holds that:

$\frac{v_{\delta}(N)}{n}-\frac{v_{\delta}(S)}{s}=\frac{(n-s) r^{2}(V-c)^{2}\left(-4 n^{3}(1+r)+4 n^{2} s(4 r+3)+n r s^{2}(9 r-4)-9 r^{2} s^{3}\right)}{4\left(4 n^{2}(1+r)+3 r^{2} s(n-s)\right)^{2}}$,

which is negative when the number of firms $n$ is sufficiently large. Thus, for each $r \in \mathbb{R}_{++}$ there exists $n_{r} \in \mathbb{N}$ such that for all $n \geq n_{r}$, the core is empty. The following table provides some values of $n_{r}$ :

\begin{tabular}{|c|ccccccccccc|}
\hline$r$ & $1 / 10$ & $1 / 5$ & $1 / 3$ & $1 / 2$ & 1 & 2 & 3 & 5 & 10 & 20 & 50 \\
\hline$n_{r}$ & 4 & 4 & 4 & 4 & 4 & 5 & 5 & 6 & 7 & 9 & 13 \\
\hline
\end{tabular}

Moreover, we have directly assumed that products are differentiated. Two other cases can be considered: when products are unrelated $(r=0)$ and when products are perfect substitutes $(r=+\infty)$. In the first case, the quantity demanded of firm $i$ 's brand only depends on its own price. Hence, the profit of a coalition does not depend on outsiders' behavior, and so the $\alpha, \beta, \gamma$ and $\delta$-characteristic functions are equal. Moreover, for every coalition $S \in 2^{N}$, the coalition profit function $\pi_{S}$ is separable:

$$
\forall x_{S} \in X_{S}, \pi_{S}\left(x_{S}\right)=\sum_{i \in S} \pi_{i}\left(x_{i}\right) .
$$

Thus, for every coalition $S \in 2^{N}$ the unique Nash equilibrium $p^{*} \in X_{N}$ of the normal form Bertrand oligopoly game $\left(N,\left(X_{i}, \pi_{i}\right)_{i \in N}\right)$ is also the unique partial agreement equilibrium under $S$. Hence, Bertrand oligopoly TU-games are additive, ${ }^{15}$ and so $(v(\{i\}))_{i \in N} \in \mathbb{R}^{n}$ is the unique core element.

In the second case, firms sell a homogeneous product. It follows that firm $i$ 's quantity demanded is positive if and only if it charges the smallest price. Since firms operate at a constant and identical marginal and average cost, for every coalition $S \in 2^{N} \backslash\{N\}$, outsiders charge prices equal to their marginal cost, ${ }^{16}$ and so coalition $S$ obtains a zero profit. By charging the monopoly price, the grand coalition obtains a non-negative profit, and we conclude that the core is equal to the set of imputations consisting of all efficient payoff vectors $\sigma \in \mathbb{R}^{n}$ such that $\sigma_{i} \geq 0$ for all $i \in N$.

\footnotetext{
${ }^{14}$ The computation of $v_{\delta}(S)$ follows from standard first-order conditions.

${ }^{15} \mathrm{~A}$ TU-game $v \in G^{N}$ is additive if for every coalition $S \in 2^{N}, v(S)=\sum_{i \in S} v(\{i\})$.

${ }^{16}$ This outsiders' behavior is consistent with the $\alpha, \beta, \gamma$ and $\delta$-characteristic functions.
} 


\section{Appendix}

Proof of Proposition 3.1: Take any coalition $S \in 2^{N}$. Define $b_{S}: X_{N \backslash S} \longrightarrow X_{S}$ the best reply function of coalition $S$ as:

$$
\forall p_{N \backslash S} \in X_{N \backslash S}, \forall p_{S} \in X_{S}, \pi_{S}\left(b_{S}\left(p_{N \backslash S}\right), p_{N \backslash S}\right) \geq \pi_{S}\left(p_{S}, p_{N \backslash S}\right)
$$

It follows from (10) that:

$$
v_{\beta}(S)=\min _{p_{N \backslash S} \in X_{N \backslash S}} \pi_{S}\left(b_{S}\left(p_{N \backslash S}\right), p_{N \backslash S}\right) .
$$

In order to compute the worth $v_{\beta}(S)$ of coalition $S$, we have to successively solve the maximization and the minimization problems defined in (3). First, for every $p_{N \backslash S} \in X_{N \backslash S}$ consider the profit maximization program of coalition $S$ :

$$
\forall p_{N \backslash S} \in X_{N \backslash S}, \max _{p_{S} \in X_{S}} \pi_{S}\left(p_{S}, p_{N \backslash S}\right) .
$$

The first-order conditions for a maximum are:

$$
\forall p_{N \backslash S} \in X_{N \backslash S}, \forall i \in S, \frac{\partial \pi_{S}}{\partial p_{i}}\left(p_{S}, p_{N \backslash S}\right)=0,
$$

and imply that the unique maximizer $b_{S}\left(p_{N \backslash S}\right)$ is given by:

$$
\forall p_{N \backslash S} \in X_{N \backslash S}, \forall i \in S, \quad b_{i}\left(p_{N \backslash S}\right)=\frac{V+(r / n) \sum_{j \in N \backslash S} p_{j}}{2(1+r(n-s) / n)}+\frac{c}{2}
$$

Then, given $b_{S}\left(p_{N \backslash S}\right) \in X_{S}$ consider the profit minimization program of the complementary coalition $N \backslash S$ :

$$
\min _{p_{N \backslash S} \in X_{N \backslash S}} \pi_{S}\left(b_{S}\left(p_{N \backslash S}\right), p_{N \backslash S}\right) .
$$

The first-order conditions for a minimum are:

$$
\forall j \in N \backslash S, \frac{\partial \pi_{S}}{\partial p_{j}}\left(b_{S}\left(p_{N \backslash S}\right), p_{N \backslash S}\right)=0,
$$

which are equivalent, for all $j \in N \backslash S$, to the following equality:

$$
\sum_{j \in N \backslash S} p_{j}=\frac{n}{r}\left(c\left(1+r \frac{(n-s)}{n}\right)-V\right) .
$$

Since for every $i \in N, X_{i}=\mathbb{R}_{+}$, it follows that any minimizer $\bar{p}_{N \backslash S} \in X_{N \backslash S}$ satisfies:

$$
\sum_{j \in N \backslash S} \bar{p}_{j}=\max \left\{0, \frac{n}{r}\left(c\left(1+r \frac{(n-s)}{n}\right)-V\right)\right\} .
$$


which proves (5). By substituting (5) into (11), we deduce that:

$$
\forall i \in S, \bar{p}_{i}=b_{i}\left(\bar{p}_{N \backslash S}\right)=\max \left\{c, \frac{V}{2(1+r(n-s) / n)}+\frac{c}{2}\right\},
$$

which proves (4) and completes the proof.

Proof of Theorem 3.4: We want to prove that Bertrand oligopoly TU-games in $\beta$ characteristic function form are convex. Proposition 3.1 implies that Bertrand oligopoly TU-games in $\beta$-characteristic function form are symmetric. It follows from (4) that the members of a coalition $S \in 2^{N}$ charge identical prices, i.e. for every $i \in S$, there exists $\bar{p}^{s} \in \mathbb{R}_{+}$such that $\bar{p}_{i}=\bar{p}^{s}$. It follows from (5) that outsiders charge an average price $\bar{p}_{[n-s]} /(n-s)$ where $\bar{p}_{[n-s]}=\sum_{j \in N \backslash S} \bar{p}_{j}$. Hence, the worth $v_{\beta}(S)$ depends only on the size $s$ of coalition $S$, i.e. there exists a function $f_{\beta}: \mathbb{N} \longrightarrow \mathbb{R}$ such that for every coalition $S \in 2^{N}$, it holds that:

$$
v_{\beta}(S)=f_{\beta}(s)=s\left(\bar{p}^{s}-c\right)\left(V-\bar{p}^{s}\left(1+r \frac{(n-s)}{n}\right)+\frac{r}{n} \bar{p}_{[n-s]}\right) .
$$

It remains to prove (1). ${ }^{17}$ Take any coalition $S \in 2^{N}$ of size $s$ such that $s \leq n-2$. First, we distinguish two cases:

- assume that $V \leq c(1+r(n-s-1) / n)$. It follows from Point 1 of Remark 3.2 that $\bar{p}^{s+1}=c$.

- assume that $V>c(1+r(n-s-1) / n)$. This implies that $V>c(1+r(n-s-2) / n)$, and it follows from Point 2 of Remark 3.2 that $\bar{p}_{[n-s-1]}=\bar{p}_{[n-s-2]}=0$.

In both cases, it holds that:

$$
\left(\bar{p}^{s+1}-c\right) \bar{p}_{[n-s-2]}=\left(\bar{p}^{s+1}-c\right) \bar{p}_{[n-s-1]}
$$

Since $\bar{p}^{s+2}$ is the unique maximizer for any coalition of size $s+2$ and from (12), it holds that:

${ }^{17}$ For a symmetric TU-game $v \in G^{N}$ where for every coalition $S \in 2^{N}, v(S)=f(s)$, condition (1) becomes:

$$
\forall S \in 2^{N}: s \leq n-2, f(s+1)-f(s) \leq f(s+2)-f(s+1)
$$




$$
\begin{aligned}
f_{\beta}(s+2) & =(s+2)\left(\bar{p}^{s+2}-c\right)\left(V-\bar{p}^{s+2}\left(1+r \frac{(n-s-2)}{n}\right)+\frac{r}{n} \bar{p}_{[n-s-2]}\right) \\
& \geq(s+2)\left(\bar{p}^{s+1}-c\right)\left(V-\bar{p}^{s+1}\left(1+r \frac{(n-s-2)}{n}\right)+\frac{r}{n} \bar{p}_{[n-s-2]}\right) \\
& =(s+2)\left(\bar{p}^{s+1}-c\right)\left(V-\bar{p}^{s+1}\left(1+r \frac{(n-s-2)}{n}\right)+\frac{r}{n} \bar{p}_{[n-s-1]}\right) \\
& =f_{\beta}(s+1)+\left(\bar{p}^{s+1}-c\right)\left(V-\bar{p}^{s+1}\left(1+r \frac{(n-2 s-3)}{n}\right)+\frac{r}{n} \bar{p}_{[n-s-1]}\right)
\end{aligned}
$$

Moreover, since $\bar{p}^{s}$ is the unique maximizer for any coalition of size $s$ and $\bar{p}_{[n-s]} \geq \bar{p}_{[n-s-1]}$, we deduce that:

$$
\begin{aligned}
f_{\beta}(s) & =s\left(\bar{p}^{s}-c\right)\left(V-\bar{p}^{s}\left(1+r \frac{(n-s)}{n}\right)+\frac{r}{n} \bar{p}_{[n-s]}\right) \\
& \geq s\left(\bar{p}^{s+1}-c\right)\left(V-\bar{p}^{s+1}\left(1+r \frac{(n-s)}{n}\right)+\frac{r}{n} \bar{p}_{[n-s]}\right) \\
& \geq s\left(\bar{p}^{s+1}-c\right)\left(V-\bar{p}^{s+1}\left(1+r \frac{(n-s)}{n}\right)+\frac{r}{n} \bar{p}_{[n-s-1]}\right)
\end{aligned}
$$

It follows from the expression of $f_{\beta}(s+1)$ and (14) that:

$$
f_{\beta}(s+1)-f_{\beta}(s) \leq\left(\bar{p}^{s+1}-c\right)\left(V-\bar{p}^{s+1}\left(1+r \frac{(n-2 s-1)}{n}\right)+\frac{r}{n} \bar{p}_{[n-s-1]}\right)
$$

We conclude from (13) and (15) that:

$$
\begin{aligned}
f_{\beta}(s+1)-f_{\beta}(s) & \leq\left(\bar{p}^{s+1}-c\right)\left(V-\bar{p}^{s+1}\left(1+r \frac{(n-2 s-1)}{n}\right)+\frac{r}{n} \bar{p}_{[n-s-1]}\right) \\
& \leq\left(\bar{p}^{s+1}-c\right)\left(V-\bar{p}^{s+1}\left(1+r \frac{(n-2 s-3)}{n}\right)+\frac{r}{n} \bar{p}_{[n-s-1]}\right) \\
& \leq f_{\beta}(s+2)-f_{\beta}(s+1),
\end{aligned}
$$

which completes the proof.

Proof of Corollary 3.6: Take any coalition $S \in 2^{N}$ and consider the coalition structure $\mathcal{P}^{S}=\{S\} \cup\{\{j\}: j \in N \backslash S\}$. It follows from (6) and (7) that a strategy profile 
$\left(p_{S}^{*}, \tilde{p}_{N \backslash S}\right) \in X_{S} \times X_{N \backslash S}$ is a partial agreement equilibrium for the normal form Bertrand oligopoly game $\left(N,\left(X_{i}, \pi_{i}\right)_{i \in N}\right)$ if and only if it is a Nash equilibrium for the normal form Bertrand oligopoly game $\left(\mathcal{P}^{S},\left(X_{T}, \pi_{T}\right)_{T \in \mathcal{P}^{S}}\right)$. By Point 1 of Proposition 3.5 we conclude that there exists a unique partial agreement equilibrium under $S$.

Proof of Theorem 3.10: Pick any $i \in N$ and any $j \in N$ such that $j \neq i$. We want to show that for any coalition $S \subseteq N \backslash\{i, j\}, v_{\gamma}(S \cup\{i\})-v_{\gamma}(S) \leq v_{\gamma}(S \cup\{i, j\})-v_{\gamma}(S \cup\{j\})$. First, assume that $S=\emptyset$. In such a case, the superadditive result in Deneckere and Davidson (1985) ensures that $v_{\gamma}(\{i\})+v_{\gamma}(\{j\}) \leq v_{\gamma}(\{i, j\})$, and so the convexity property holds for $S=\emptyset$.

Then, assume that $S \neq \emptyset$. For any $S \subseteq N$ such that $s \geq 1$, it follows from (8) and (9) that the worth $v_{\gamma}(S)$ depends only on the size $s$ of coalition $S$, i.e. there exists a function $f_{\gamma}: \mathbb{N} \longrightarrow \mathbb{R}$ such that for every coalition $S \in 2^{N}$, it holds that:

$$
v_{\gamma}(S)=f_{\gamma}(s)=\frac{s(V-c)^{2}(2 n(1+r)-r)^{2} n(n+r(n-s))}{\left(4 n^{2}+6 n^{2} r-2 n r s+2 n^{2} r^{2}-r^{2} s^{2}-2 n r-2 n r^{2}+2 r^{2} s-n r^{2} s\right)^{2}} .
$$

Since mergers are increasingly profitable (Deneckere and Davidson 1985), the function $f_{\gamma}$ is increasing. In order to prove the convexity property (1), we want to show that for any $s \geq 1$, the second derivative of $f_{\gamma}$ is non-negative. This second derivative is given by:

$$
\frac{d^{2} f_{\gamma}}{d s^{2}}(s)=\frac{A}{B(s)}\left(r^{3}(n-s) g_{1}(s)+r^{2} g_{2}(s)+r 4 n^{2} g_{3}(s)-8 n^{3}+12 n^{3} s\right),
$$

where:

$$
\begin{aligned}
A & =2(V-c)^{2}(2 n(1+r)-r)^{2} n r^{2} \geq 0 ; \\
B(s) & =\left(4 n^{2}+6 n^{2} r-2 n r s+2 n^{2} r^{2}-r^{2} s^{2}-2 n r-2 n r^{2}+2 r^{2} s-n r^{2} s\right)^{4} \geq 0 ; \\
g_{1}(s) & =3 s^{3}+9 n^{2} s-4 n^{2}-8 n s+4 n-3 n s^{2} \\
g_{2}(s) & =6 n s^{3}+\left(8 n-36 n^{2}\right) s^{2}+\left(33 n^{3}-4 n\right) s-16 n^{3}+8 n^{2} \\
g_{3}(s) & =-6 s^{2}+(9 n+1) s+1-5 n .
\end{aligned}
$$

It remains to show that the functions $g_{1}, g_{2}$ and $g_{3}$ are non-negative. 


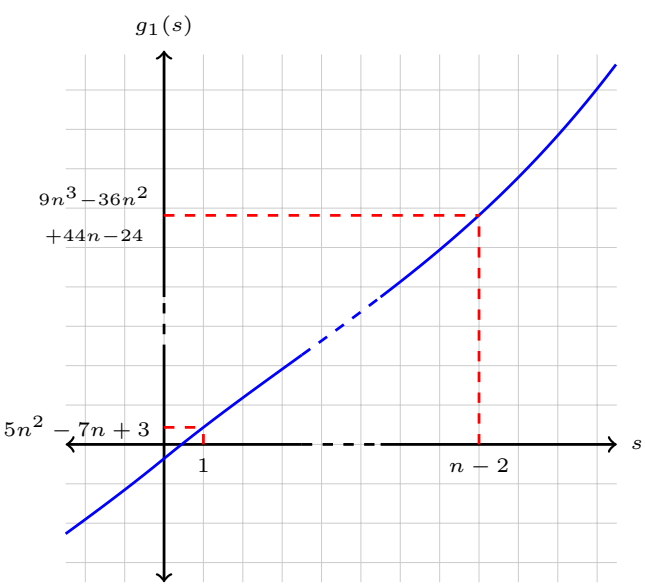

Figure 1

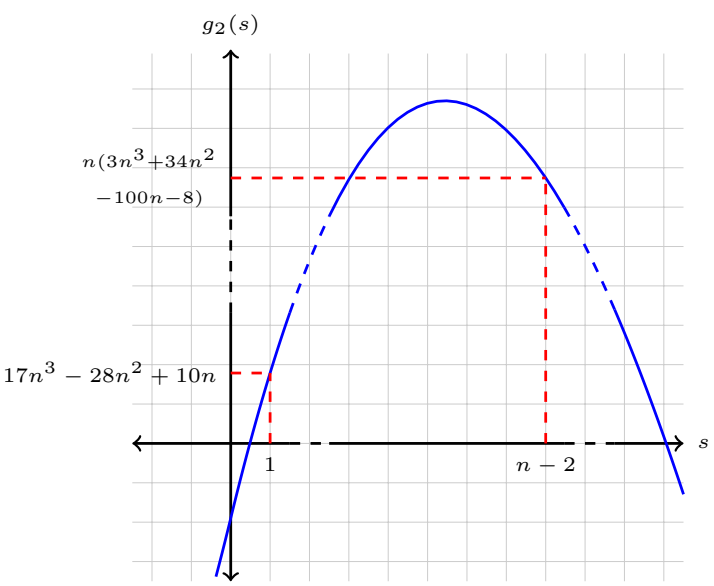

Figure 2

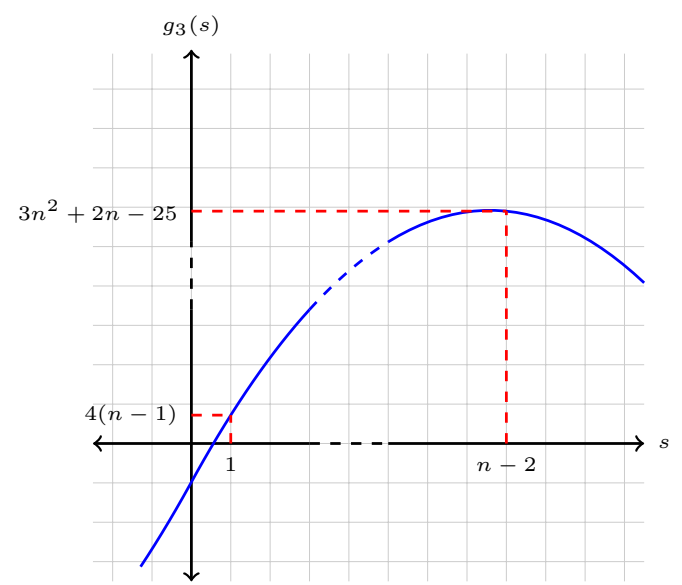

Figure 3

First, the function $g_{1}$ is increasing with respect to $s$ so that its minimum is $g_{1}(1)=5 n^{2}-7 n+$ $3 \geq 0$ as illustrated by Figure 1 . Then, the function $g_{2}$ is also a polynomial function of degree 3 represented by Figure 2 . We can verify that $d g_{2}(s) / d s=18 n s^{2}+2\left(8 n-36 n^{2}\right) s+33 n^{3}-4 n$ has two real roots $s_{1}$ and $s_{2}$ such that:

$$
1 \leq s_{1}=2 n-\frac{4}{9}-\frac{1}{18} \sqrt{702 n^{2}-576 n+136} \leq n,
$$

and

$$
n \leq s_{2}=2 n-\frac{4}{9}+\frac{1}{18} \sqrt{702 n^{2}-576 n+136} .
$$

So, $g_{2}$ is increasing on $\left[1 ; s_{1}\right]$ and decreasing on $\left[s_{1} ; n\right]$. Moreover, $g_{2}(1) \geq 0$ and $g_{2}(n) \geq 0$ implying that $g_{2}$ is non-negative. Finally, the function $g_{3}$ is a polynomial function of degree 2 represented by Figure 3 and we can verify that it is non-negative for any $s \in\{1, \ldots, n\}$ and $n \geq 3$, which concludes the proof. 


\section{References}

Aumann, R. (1959). Acceptable points in general cooperative n-person games, in: Tucker, luce (eds.), Contributions to the theory of games IV. Annals of Mathematics Studies Vol. 40, Princeton University Press, Princeton.

Bloch, F. (1995). Endogenous structures of association in oligopolies. The RAND Journal of Economics 26(3), 537-556.

Chander, P. and H. Tulkens (1997). The core of an economy with multilateral environmental externalities. International Journal of Game Theory 26, 379-401.

Deneckere, R. and C. Davidson (1985). Incentives to form coalitions with Bertrand competition. The RAND Journal of Economics 16, 473-486.

Driessen, T. S., D. Hou, and A. Lardon (2011). Convexity and the Shapley value in Bertrand oligopoly TU-games with Shubik's demand functions. Working paper, University of Saint-Etienne.

Driessen, T. S., D. Hou, and A. Lardon (2017). Stackelberg Oligopoly TU-Games: Characterization and Nonemptiness of the Core. International Game Theory Review 19:(1750020)1-16.

Hart, S. and M. Kurz (1983). Endogenous formation of coalitions. Econometrica 51(4), 1047-1064.

Huang, C.-Y. and T. Sjöström (2003). Consistent solutions for cooperative games with externalities. Games and Economic Behavior 43(2), 196-213.

Julien, L. A. (2017). On noncooperative oligopoly equilibrium in the multiple leaderfollower game. European Journal of Operational Research 256(2), 650-662.

Kaneko, M. (1978). Price oligopoly as a cooperative game. International Journal of Game Theory 7(3), 137-150.

Lardon, A. (2012). The $\gamma$-core in Cournot oligopoly TU-games with capacity constraints. Theory and Decision 72, 387-411.

Lardon, A. (2014). A partial characterization of the core in Bertrand oligopoly TU-games with transferable technologies. Working Paper, Université Côte d'Azur, CNRS, GREDEG, France.

Lardon, A. (2017). Endogenous interval games in oligopolies and the cores. Annals of Operations Research 248, 345-363.

Lekeas, P. V. and G. Stamatopoulos (2014). Cooperative oligopoly games with boundedly rational firms. Annals of Operations Research 223(1), 255-272.

Levenstein, M. C. (1997). Price Wars and the Stability of Collusion: A Study of the PreWorld War I Bromine Industry. The Journal of Industrial Economics 45(2), 117-137.

Norde, H., K. H. Pham Do, and S. Tijs (2002). Oligopoly games with and without transferable technologies. Mathematical Social Sciences 43, 187-207.

Shapley, L. (1955). Markets as cooperative games. RAND Corporation Paper P-629, 1-5. 
Shapley, L. S. (1971). Cores of convex games. International Journal of Game Theory 1, 11-26.

Shubik, M. (1980). Market structure and behavior. Cambridge: Harvard University Press.

Zhao, J. (1999a). A necessary and sufficient condition for the convexity in oligopoly games. Mathematical Social Sciences 37, 189-204.

Zhao, J. (1999b). A $\beta$-Core Existence Result and Its Application to Oligopoly Markets. Games and Economic Behavior 27, 153-168. 